\title{
ARTICLE
}

\section{Intestinal epithelium-derived BATF3 promotes colitis- associated colon cancer through facilitating CXCL5-mediated neutrophils recruitment}

\author{
Y. Lin ${ }^{1}$, L. Cheng ${ }^{1}$, Y. Liu ${ }^{1}$, Y. Wang ${ }^{1}$, Q. Wang ${ }^{1}$, H. L. Wang ${ }^{1}$, G. Shi ${ }^{1}$, J. S. Li ${ }^{1}$, Q. N. Wang ${ }^{1}$, Q. M. Yang ${ }^{1}$, S. Chen ${ }^{1}$, X. L. Su ${ }^{1}$, Y. Yang ${ }^{1}$, \\ M. Jiang ${ }^{2}$, X. Hu ${ }^{3}$, P. Fan ${ }^{3}$, C. Fang ${ }^{4}$, Z. G. Zhou ${ }^{4}$, L. Dai ${ }^{1}$ and H. X. Deng (iD ${ }^{1}$
}

Inflammation is a critical player in the development and progression of colon cancer. Basic leucine zipper transcription factor ATFlike 3 (BATF3) plays an important role in infection and tumor immunity through regulating the development of conventional type 1 dendritic cells (CDC1s). However, the function of BATF3 in colitis and colitis-associated colon cancer (CAC) remains unclear. Here, BATF3 wild-type and knockout mice were used to construct an AOM/DSS-induced CAC model. In addition, DSS-induced chronic colitis, bone marrow cross-transfusion (BMT), neutrophil knockout, and other animal models were used for in-depth research. We found that BATF3 deficiency in intestinal epithelial cells rather than in CDC1s inhibited CAC, which was depended on inflammatory stimulation. Mechanistically, BATF3 directly promoted transcription of CXCL5 by forming a heterodimer with JunD, and accelerated the recruitment of neutrophils through the CXCL5-CXCR2 axis, ultimately increasing the occurrence and development of CAC. Tissue microarray and TCGA data also indicated that high expression of BATF3 was positively correlated with poor prognosis of colorectal cancer and other inflammation-related tumors. In summary, our results demonstrate that intestinal epithelial-derived BATF3 relies on inflammatory stimulation to promote CAC, and BATF3 is expected to be a novel diagnostic indicator for colitis and CAC.

Mucosal Immunology (2021) 14:187-198; https://doi.org/10.1038/s41385-020-0297-3

\section{INTRODUCTION}

Colorectal cancer (CRC) can be effectively detected and prevented before malignant transformation. ${ }^{1}$ However, most cases are diagnosed in an advanced stage and have poor overall survival, making CRC the second most frequent cause of cancer-related deaths. $^{2}$ The etiology of CRC is multifactorial, including mutations, inflammatory bowel disease, gut microbiota, and diet. ${ }^{3}$ Among these, inflammation is a critical player in the development, progression, and metastasis of both CAC and sporadic CRC. ${ }^{4}$ Therefore, understanding the pathogenesis of colitis promoting CAC will provide novel therapy strategies for CRC.

The main pathological feature of CRC involves dysregulated intestinal homeostasis, characterized by mucosal damage and microbiota translocation, which contribute to colon carcinogenesis by recruiting proinflammatory immune cells, producing tumor-promoting cytokines, and establishing a tumor immune microenvironment. ${ }^{5}$ Among infiltrating immune cells, neutrophils are the most abundant involved in innate immunity, ${ }^{6,7}$ and their numbers are significantly increased in patients and mice with colitis or CRC. ${ }^{8,9}$ Neutrophils play an important regulatory role in tumor initiation, proliferation, immune regulation, angiogenesis, and metastasis in various tumors. ${ }^{6}$ Recruitment of neutrophils to the tumor location is regulated by interactions between the chemokines CXCL1, CXCL2, CXCL5, CXCL7, CXCL12, and chemokine receptors CXCR1 and CXCR2. Inhibition of CXCR2 or clearance of neutrophils can significantly impair tumor development and progression. ${ }^{9,10}$ It is widely accepted that neutrophils contribute to cancer immune evasion by suppressing the functions of T and NK cells. ${ }^{11}$ However, the mechanisms by which these chemokines increase and neutrophils promote CAC progression remain to be explored.

Basic leucine zipper transcription factor ATF family contains BATF, BATF2, and BATF3, which competes with c-Fos to form a heterodimer with c-Jun. ${ }^{12}$ It has been demonstrated that BATF is highly expressed in colon cancer and promotes CAC by regulating Th17 cells, ${ }^{13}$ whereas BATF2 demonstrates low expression in CRC and plays an important role in inhibiting angiogenesis in colon cancer. ${ }^{14}$ BATF3 is crucial to the development of CD4 ${ }^{-} \mathrm{CD} 8 \mathrm{a}^{+} \mathrm{DCs}$

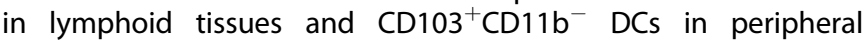
tissues. ${ }^{15,16}$ During infection and tumor immunity, CDC1s regulated by BATF3 are stimulated to secrete interleukin 12 (IL-12), thereby stimulating $T$ and NK cells to secrete interferon $\gamma$ (IFN $\gamma$ ), thus inhibiting tumors and infection. ${ }^{15,17,18}$ In addition to regulating the development of $\mathrm{cDC} 1 \mathrm{~s}, \mathrm{BATF} 3$ also acts as a

\footnotetext{
${ }^{1}$ State Key Laboratory of Biotherapy and Cancer Center, West China Hospital, Sichuan University and Collaborative Innovation Center for Biotherapy, Chengdu 610041 Sichuan, China; ${ }^{2}$ Department of Medical Oncology, Cancer Center and State Key Laboratory of Biotherapy, West China Hospital of Sichuan University, Chengdu, Sichuan, China; ${ }^{3}$ West China Biobanks, Department of Clinical Research Management and West China-Liverpool Biomedical Research Centre, West China Hospital, Sichuan University, Chengdu, Sichuan, China and ${ }^{4}$ Department of Gastrointestinal Surgery, West China Hospital and State Key Laboratory of Biotherapy, Sichuan University, Chengdu, Sichuan, China Correspondence: M. Jiang (307048372@qq.com) or L. Dai (dailei2016@scu.edu.cn) or H. X. Deng (denghongx@scu.edu.cn)

These authors contributed equally: Y. Lin, L. Cheng
}

Received: 13 February 2020 Revised: 24 April 2020 Accepted: 1 May 2020

Published online: 28 May 2020 
proto-oncogene to promote the proliferation and invasion of lymphoma. ${ }^{19,20}$ Previous work has demonstrated that BATF3 is upregulated and associated with poor prognosis in $\mathrm{CRC}_{i}^{21}$ however, the functional role of BATF3 in colitis and CAC has not been reported.

In this study, we found that BATF3 deficient in intestinal epithelial cells could suppress CAC depending on reducing recruitment of neutrophils. Mechanically, BATF3 transcriptively activated chemokine CXCL5 expression in epithelial under inflammatory stimuli leading to recruitment of $\mathrm{CXCR2}^{+}$neutrophils. Collectively, the results of our study point to a novel diagnostic indicator for colitis and CAC, as well as for other inflammation-associated cancers.

\section{RESULTS}

Intestinal epithelial-derived BATF3 promotes the development of CAC

To investigate the functional role of BATF3 in CAC, an AOM/DSS model was established in WT and BATF3 ${ }^{-1-}$ mice (Fig. S1A-C). The survival time of $\mathrm{BATF}^{-1-}$ mice was significantly prolonged (Fig. 1a). High-resolution mini-endoscopy imaging and macroscopic images showed that BATF3 ${ }^{-1-}$ observably suppressed the size and number of tumor (Fig. 1b, c). Statistical analysis found that the colon length of BATF ${ }^{-1-}$ mice was longer, the number of tumors was reduced (Fig. 1d), and the tumor area and tumor load were smaller (Fig. S1D). H\&E staining indicated that the colon tissues of BATF3 ${ }^{-1-}$ mice had fewer tumor areas, less colon injury, and less immune cell infiltration (Fig. 1e). Further, we detected decreased proliferation, increased apoptosis, and decreased expression of inflammatory factors in the colon tissues of $\mathrm{BATF}^{-1-}$ mice (Fig. 1f, g, S1E). Stool score and bleeding score were also lower in BATF3 ${ }^{-1-}$ mice (Fig. S1F). As reported, ${ }^{21}$ BATF3 was highly expressed in human CRC tissues (Fig. S2A, B). We found that BATF3 was also highly expressed in mouse CAC tissues (Fig. S2C-E), and BATF3 was mainly expressed in epithelial cells of human and mouse colon cancer (Fig. 1h, i). Our results also confirmed the significant reduction of $\mathrm{CDC} 1 \mathrm{~s}$ in $\mathrm{BATF}^{-1-}$ mice $^{15,16}$ (Fig. S3A, B), but there was no significant difference in $\mathrm{CD}_{103}{ }^{+} \mathrm{CD} 11 \mathrm{~b}^{-}$DCs and DNGR- ${ }^{+} \mathrm{CD}_{103}{ }^{+}$DCs between untreated and AOM/DSS-treated mice ${ }^{22,23}$ (Fig. 1j, S3C). Furthermore, BATF3 deficiency had limited effects on colon development, proliferation activity, apoptosis activity, and inflammatory cytokine expression under normal conditions ${ }^{16}$ (Fig. S3D-H). Thus, we speculated that BATF3 may play a crucial role in the AOM/DSS process through other mechanisms rather than regulating $\mathrm{CDC} 1 \mathrm{~s}$. To address this, BMT mice were constructed for the CAC model. We found that tumor number and area were significantly reduced in $\mathrm{BATF}^{-1-}$ receptor mice (Fig. $1 \mathrm{k}, \mathrm{I}$ ), indicating that BATF3 knockout inhibited colon cancer due to the deletion of BATF3 gene in non-immune cells. Above all, BATF3 deficiency in intestinal epithelial cells attenuates AOM/DSS-induced CAC progression.

\section{BATF3 deficiency suppresses CAC depends on inflammation} In the AOM/DSS-mediated primary cancer model, AOM-mediated mutation and DSS-mediated inflammation have synergistic effects in the development of CAC. ${ }^{24}$ To address whether inflammation is necessary for BATF3 to promote CAC, we evaluated a colitisindependent colon cancer model (Fig. S4A). Our results showed no dramatic difference in tumor incidence (Fig. 2a-c). Further statistical analysis confirmed there were no significant differences in colon length (Fig. S4B). H\&E staining and qPCR also showed no notable differences in tumor area and inflammation (Fig. 2d, e). In addition, WT and $\mathrm{BATF}^{-1-}$ mice were administered $2 \%$ DSS to induce chronic colitis (Fig. S4C). Representative mini-endoscopic images indicated that $\mathrm{BATF}^{-/-}$mice developed mild colitis (Fig. 2f). Macroscopic images and statistical analysis found that the colon length of $\mathrm{BATF}^{-1-}$ mice was much longer in the chronic colitis model (Fig. $2 \mathrm{~g}, \mathrm{~h}$ ), and the spleen of BATF ${ }^{-1-}$ mice was much smaller (Fig. S4D). Compared with WT mice, body weight reduction in $\mathrm{BATF}^{-1-}$ mice was significantly less (Fig. S4E), and stool score and bleeding scores were lower (Fig. 2i). H\&E staining also indicated that colon tissues of BATF $3^{-1-}$ mice had smaller areas of tissue destruction with altered crypt structure accompanied by less immune cell infiltration (Fig. 2j). Further, we found decreased expression of inflammatory factors and proliferation (Fig. $2 \mathrm{k}, \mathrm{l}$ ) in the colon tissues of BATF $3^{-1-}$ mice. Taken together, these results indicate that inflammation is required for BATF3 to promote colon cancer.

\section{BATF3 deficiency inhibits CAC depends on recruitment of} neutrophils

The mechanism by which BATF3 promotes the occurrence and development of CAC remains unclear. As demonstrated by $\mathrm{H} \& \mathrm{E}$ staining in the AOM/DSS and DSS models, infiltration of immune cells in colon tissues of BATF $3^{-1-}$ mice was reduced. Consistently, flow cytometry revealed that BATF $3^{-1-}$ mice had fewer immune cells $\left(C D 45^{+}\right)$in the colonic lamina propria in the AOM/DSS model (Fig. 3a). To examine whether BATF3 was involved in immune cell infiltration, we analyzed immune cell profiles in the colonic lamina propria at the end of the AOM/DSS treatment, found that there were many neutrophils $\left(\mathrm{CD} 11 \mathrm{~b}^{+} \mathrm{Ly}_{6 \mathrm{G}}{ }^{+}\right)$in WT mice, and they were significantly decreased in BATF $3^{-1-}$ mice (Fig. 3b). Further, flow cytometry detected neutrophils in colonic lamina propria at 0,21 , and 70 days of the AOM/DSS model, confirming that neutrophils were significantly increased in the course of CAC, but that there were significantly fewer neutrophils in BATF3 ${ }^{-1-}$ mice (Fig. 3c), which were also confirmed by IF staining (Fig. 3d). Similarly, neutrophils were significantly decreased in BATF3 ${ }^{-/-}$ mice in the chronic colitis model (Fig. S5A). However, there was no difference in neutrophils in the colon, bone marrow, blood, and liver between WT and BATF3 mice under normal conditions (Fig. 3c, S5B), indicating that BATF3 does not affect the development of neutrophils, but affects their recruitment instead. We also found that neutrophils were significantly reduced in BATF $^{-1-}$ receptor mice at the end of the AOM/DSS treatment (Fig. 3e). The above results suggest that BATF3 expressed by epithelial cells plays an important role in promoting neutrophil recruitment. Neutrophils have been reported to increase significantly and play an important role in colitis and colon cancer tissues in patients and mice. ${ }^{6,8,9}$ It is widely accepted that neutrophils inhibit the infiltration and function of $\mathrm{T}$ cells. ${ }^{25,26}$ Our flow cytometry results also showed decreased IL-17A ${ }^{+} \mathrm{CD} 4^{+}$ $\mathrm{T}$ cells and increased $\mathrm{CD}^{+}{ }^{+} \mathrm{IFN} \gamma^{+} \mathrm{T}$ cells in the colon tissues of $\mathrm{BATF}^{-1-}$ mice treated with AOM/DSS (Fig. 3f, g).

To determine whether BATF3 promotes the progression of CAC by inhibiting the recruitment of neutrophils, neutrophils were depleted in WT and BATF3 ${ }^{-1-}$ mice by injecting anti-mouse Ly6G antibody $1 A 8$ during the modeling process. ${ }^{27}$ We found that neutrophil deletion suppressed the tumor number and area in WT mice, but had little effect on BATF3 ${ }^{-/-}$mice (Fig. 3h, i, S5C-E). These results demonstrated that BATF3 deficiency inhibition CAC is dependent on recruitment of neutrophils.

BATF3 deficiency restrains CAC by inhibiting the secretion of CXCL5 in intestinal epithelial cells

To investigate how BATF3 promotes chemotaxis of neutrophils, we examined the expression of related proteins using inflammation antibody array. Among the differentially expressed proteins in colon tissues under the AOM/DSS treatment, the expression of CXCL5 was highest in WT mice and significantly decreased in BATF3 $^{-1-}$ mice (Fig. 4a), which plays an important role in recruiting neutrophils., ${ }^{9,28,29}$ Similarly, ELISA showed that CXCL5 was significantly increased in WT mice after treatment with AOM/ DSS, while BATF3 ${ }^{-1-}$ mice showed much lower levels (Fig. 4b). 


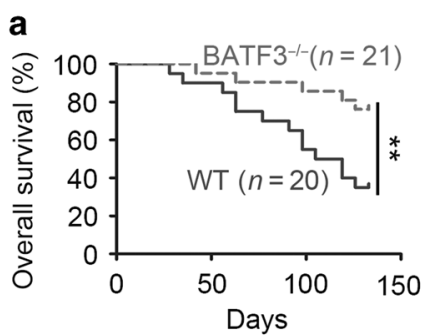

b

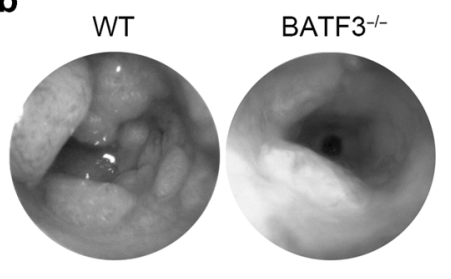

C

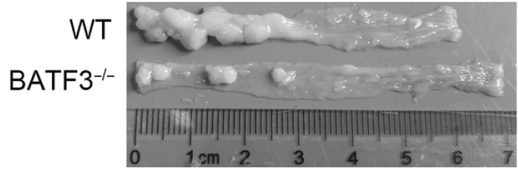

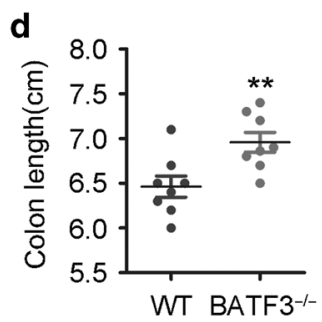

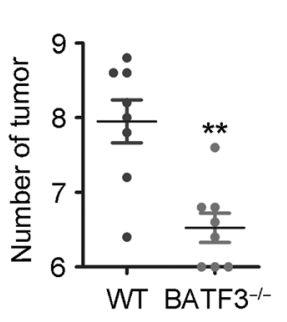

e

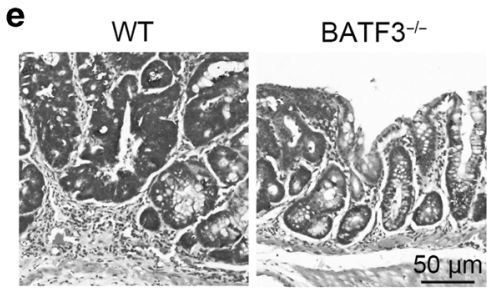

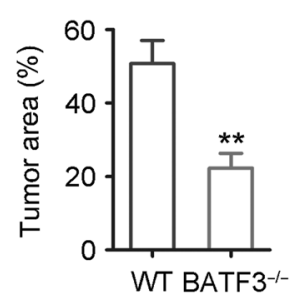

f

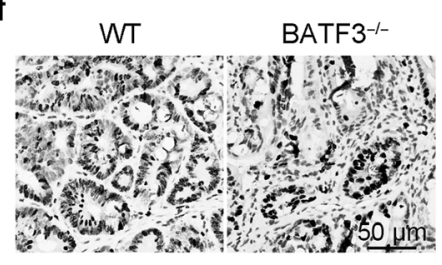

h

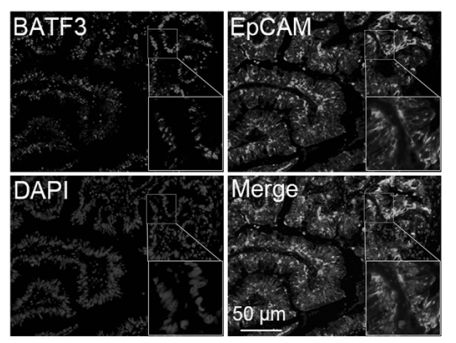

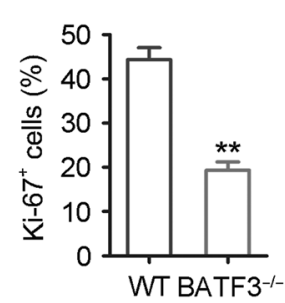

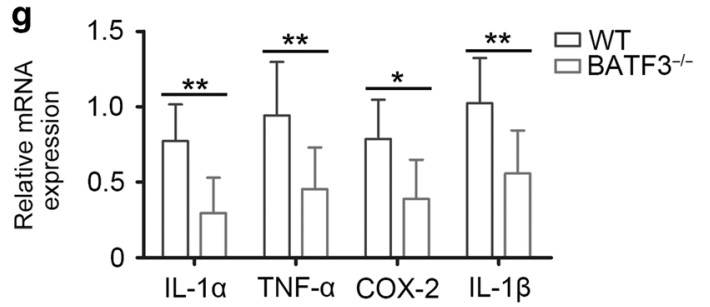

i

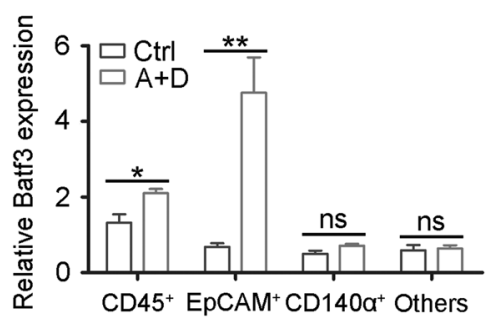

j

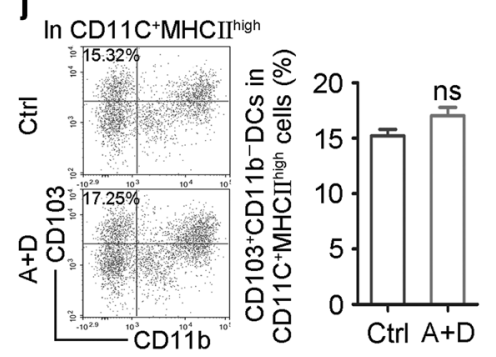

k

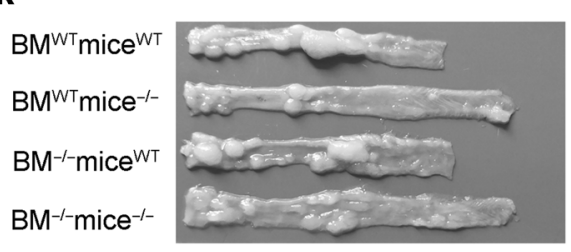

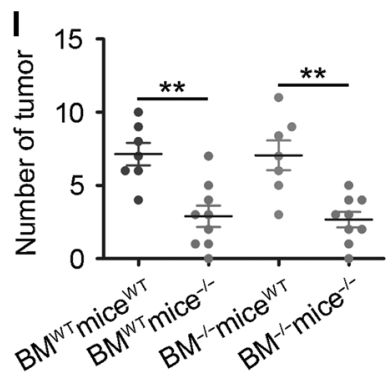

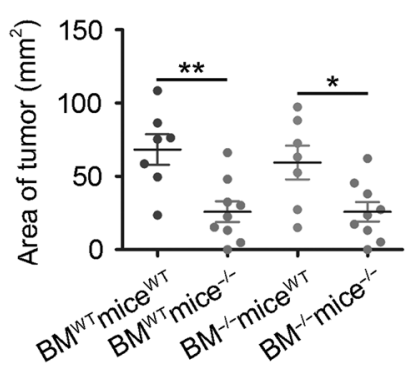

Fig. 1 Intestinal epithelial-derived BATF3 promotes the development of CAC. a Overall survival curve of WT and BATF3 ${ }^{-1-}$ mice in the AOM/DSS model (log-rank test). Representative mini-endoscopy images (b) and images of colon tissues (c) at the end of the AOM/DSS treatment. d Statistical analysis of colon length and tumor number in WT and BATF3 ${ }^{-1-}$ mice $(n=8)$. e H\&E stained images and tumor area statistics of colon tissues $(n=7)$. f Proliferation marker Ki-67 staining of colon tissues by IHC and quantification of Ki-67 ${ }^{+}$cells $(n=3)$. $\mathbf{g}$ qPCR analysis of the relative expression of inflammatory factors (IL- $1 \alpha$, TNF- $\alpha$, COX-2 and IL-1 $\beta$ ) in colon tissues $(n=4)$. h Representative IF-stained images of BATF3 and EpCAM in human malignant colon tissues, counterstained with DAPI. $\mathbf{i}$ qPCR analysis of BATF3 relative expression in various cell types screened by flow cytometry from untreated or AOM/DSS-treated colon tissues $\left(n=4 ; \mathrm{EpCAM}^{+}\right.$refers to intestinal epithelial cells; $C D 45^{+}$refers to intestinal immune cells; CD140 $\alpha^{+}$refers to intestinal fibroblasts; EpCAM ${ }^{-} \mathrm{CD}_{45}{ }^{-} \mathrm{CD} 140 \alpha^{-}$refers to the other cells). $\mathbf{j}$ Flow cytometry analysis of the $\mathrm{CD}_{103}{ }^{+} \mathrm{CD} 11 \mathrm{~b}^{-}$DCs in untreated or AOM/DSS-treated colon tissues $(n=4)$. $\mathbf{k}$ Representative images of colon tissues from bone marrow chimeric mice at the end of the AOM/DSS treatment $\left(\mathrm{BM}^{-/-}\right.$mice ${ }^{\mathrm{WT}}$ means that BATF $3^{-/-} \mathrm{BM}$ was injected back into BATF $3^{\mathrm{WT}}$ mice, and the others were in sequence). I Statistical analysis of tumor number and tumor area $(n \geq 7)$. All data are representative of three independent experiments. See also Figs. S1-S3. 


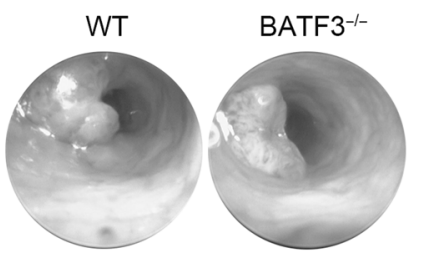

d

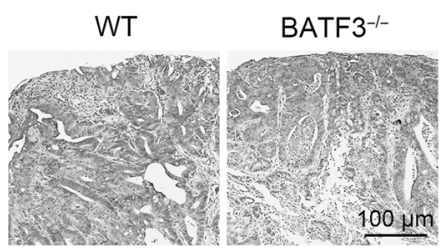

f

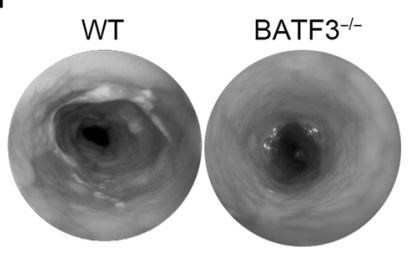

b

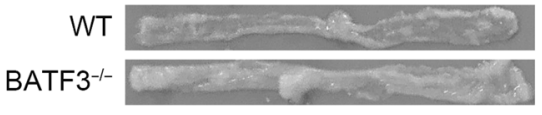

C

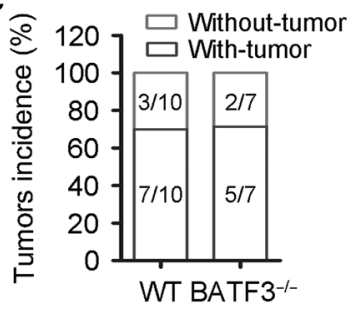

e

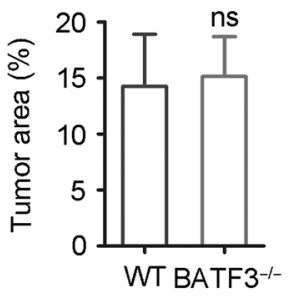

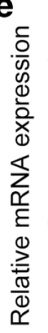

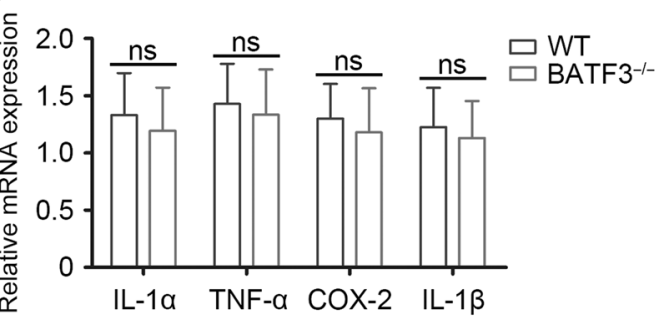

g

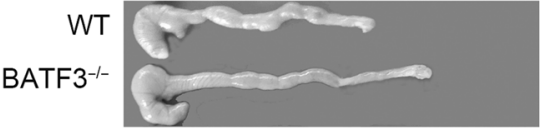

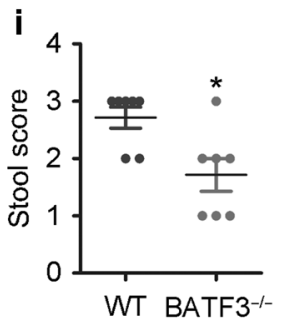
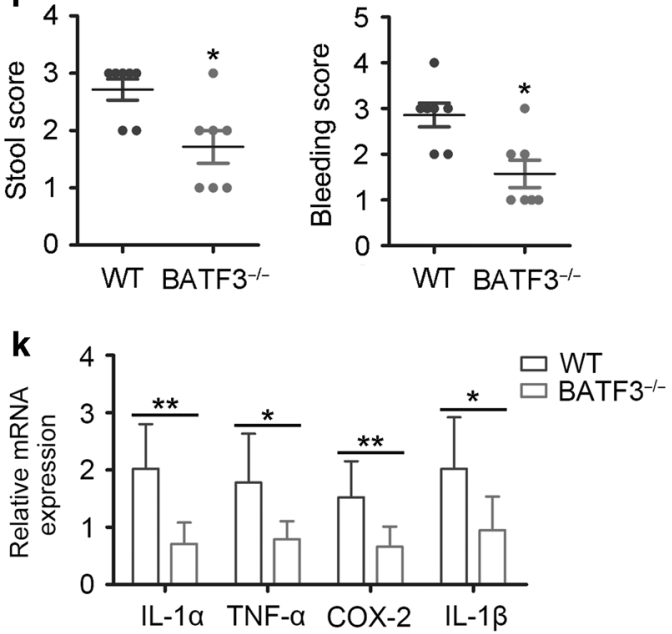

j
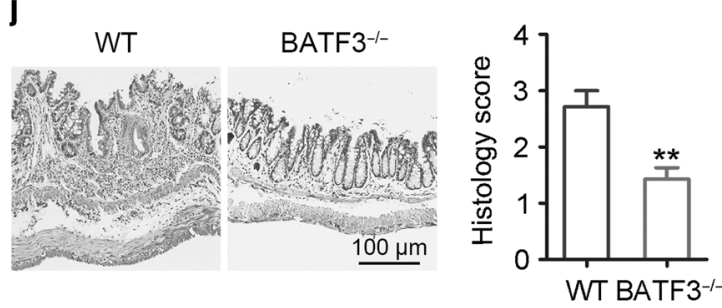

I

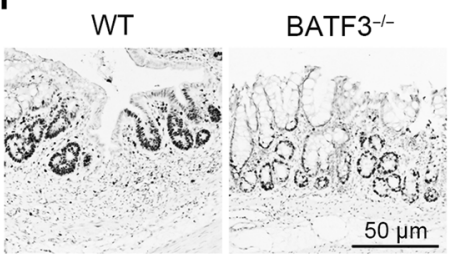

Fig. 2 BATF3 deficiency suppresses CAC depends on inflammation. Representative mini-endoscopy images (a) and images of colon tissues (b) at the end of 6XAOM treatment. c Statistical analysis of tumor incidence $(n \geq 7)$. d H\&E-stained images and tumor area statistics of colon tissues treated by AOM ( $n=7)$. e qPCR analysis of the relative expression of inflammatory factors in colon tissues $(n=7)$. Representative miniendoscopy images $(\mathbf{f})$ and images of colon tissues $(\mathbf{g})$ at the end of DSS treatment. $\mathbf{h}$ Statistical analysis of colon length of WT and BATF3 ${ }^{-1-}$ mice at the end of the DSS treatment $(n=7)$. i Statistical analysis of stool score and bleeding score $(n=7$, Mann-Whitney test). $\mathbf{j}$ H\&E stained images and corresponding histological score statistics of colon tissues ( $n=7$, Mann-Whitney test). $\mathbf{k}$ Ki- 67 staining of colon tissues by IHC and quantification of $\mathrm{Ki}-67^{+}$cells $(n=3)$. I qPCR analysis of the relative expression of several inflammatory factors $(n=7)$. All data are representative of three independent experiments. See also Fig. S4.

Other chemokines ${ }^{30}$ promoting neutrophil chemokines showed no significant difference (Fig. S6A, B). Consistently, CXCL5 was also significantly reduced in BATF3 ${ }^{-1-}$ mice in the chronic colitis model (Fig. S6C). Furthermore, ELISA showed that CXCL5 expression was significantly reduced in $\mathrm{BATF}^{-1-}$ receptor mice at the end of the AOM/DSS treatment (Fig. S6D). qPCR and IF staining both confirmed that CXCL5 was mainly expressed in epithelial cells in the AOM/DSS model (Fig. 4c, e). CXCL5 relative expression was also much lower in colon organoids of $\mathrm{BATF}^{-1-}$ mice (Fig. $4 \mathrm{~d}$, S6E). Neutrophil chemotactic receptors mainly include CXCR1 and
CXCR2. ${ }^{31}$ We found that CXCR2 was significantly lower in colon tissues of $\mathrm{BATF}^{-1-}$ mice, but CXCR1 showed no marked difference in the AOM/DSS model (Fig. 4f, S6F). qPCR and flow cytometry further confirmed that CXCR2 was specifically expressed in neutrophils (Fig. 4g, h). As reported, CXCL5 recruited neutrophils via CXCR2 in vivo and in vitro chemotactic experiments. ${ }^{7,9}$ Based on the above results, we speculate that BATF3 regulates the secretion of CXCL5 in intestinal epithelial cells, thereby recruiting neutrophils through CXCR2 to promote CAC. 

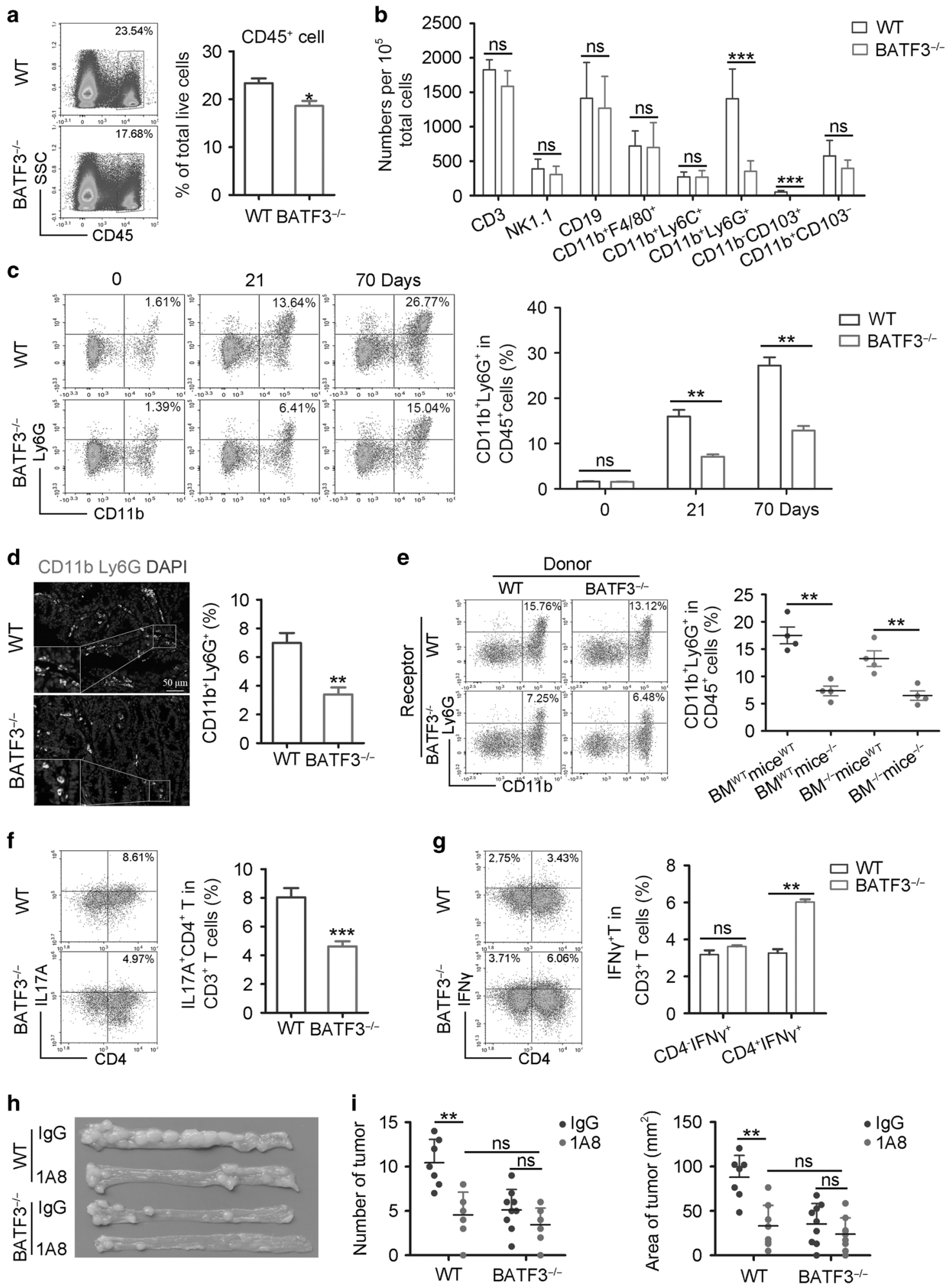

Fig. 3 BATF3 deficiency inhibits CAC depends on recruitment of neutrophils. a Flow cytometry analysis of CD45 ${ }^{+}$cells in colon tissues at the end of the AOM/DSS treatment $(n=5)$. $\mathbf{b}$ Flow cytometry analysis of various types of immune cells in colon tissues at the end of AOM/DSS treatment $(n=5)$. c Flow cytometry analysis CD11 b ${ }^{+}$Ly6G ${ }^{+}$neutrophils in colon tissues at 0,21 , and 70 days of the AOM/DSS model $(n=3)$. d Representative IF images and quantification of neutrophils based on CD11b and Ly6G staining in colon tissues at the end of AOM/DSS treatment $(n=4)$. e Flow cytometry analysis of $C D 11 \mathrm{~b}^{+} \mathrm{Ly} 6 \mathrm{G}^{+}$in colon tissues of bone marrow chimeric mice at the end of AOM/DSS treatment $(n=4)$. (f) Flow cytometry analysis of IL17A ${ }^{+} \mathrm{CD} 4^{+}$T cells in colon tissues of WT and BATF3 ${ }^{-1-}$ mice at the end of AOM/ DSS treatment $(n=3)$. $\mathbf{g}$ Flow cytometry analysis of INF $\gamma^{+}$T cells in colon tissues $(n=3)$. $\mathbf{h}$ Representative images of colon tissues from IgG or anti-mouse Ly6G antibody (1A8)-injected WT and BATF3 ${ }^{-1-}$ mice at the end of AOM/DSS treatment. $\mathbf{i}$ Statistical analysis of colon tumor number and tumor area $(n \geq 7)$. All data are representative of three independent experiments. See also Fig. S5. 
a

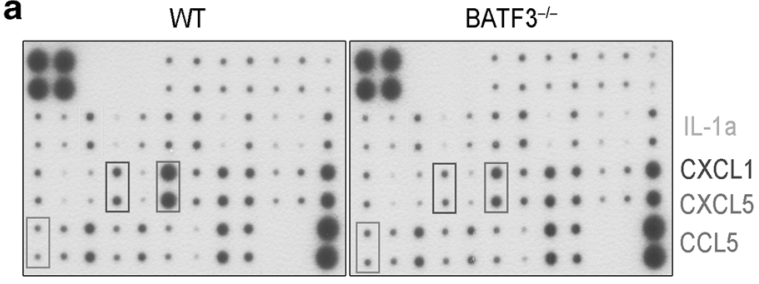

b

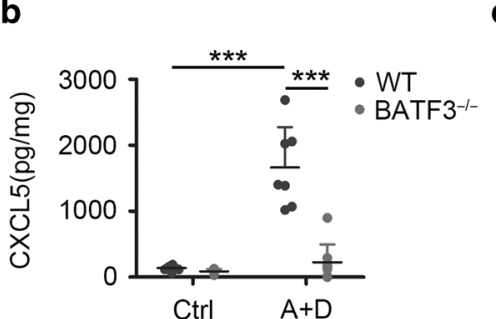

c.

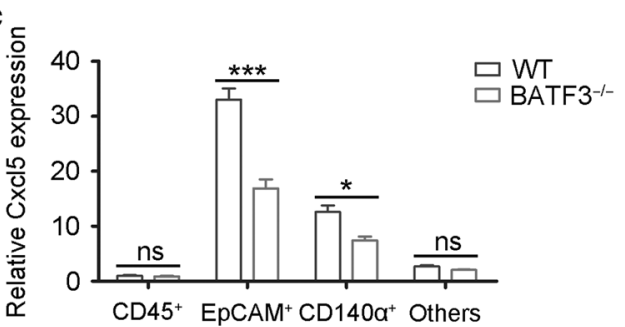

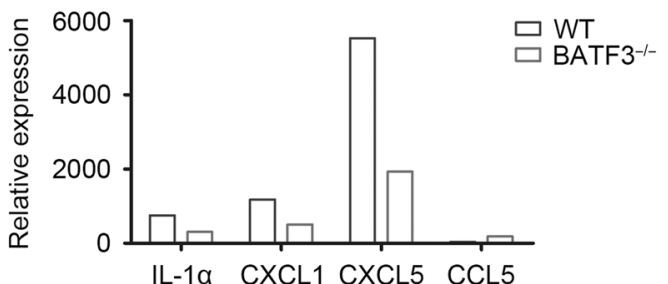

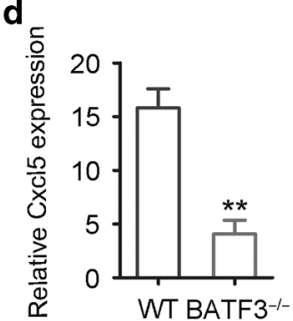

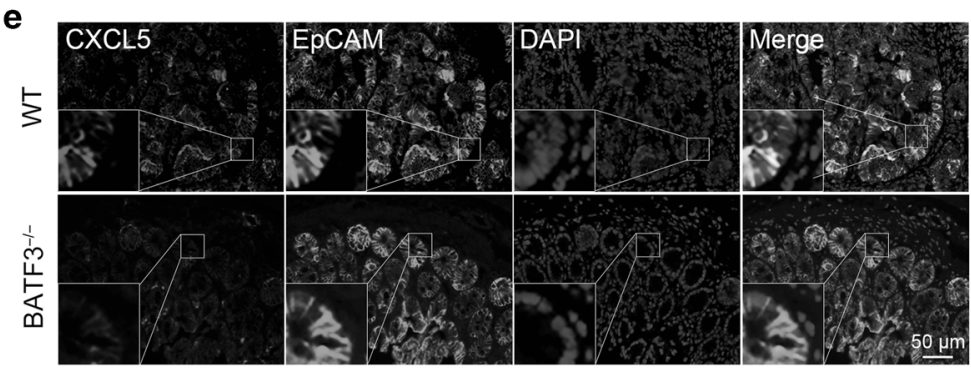
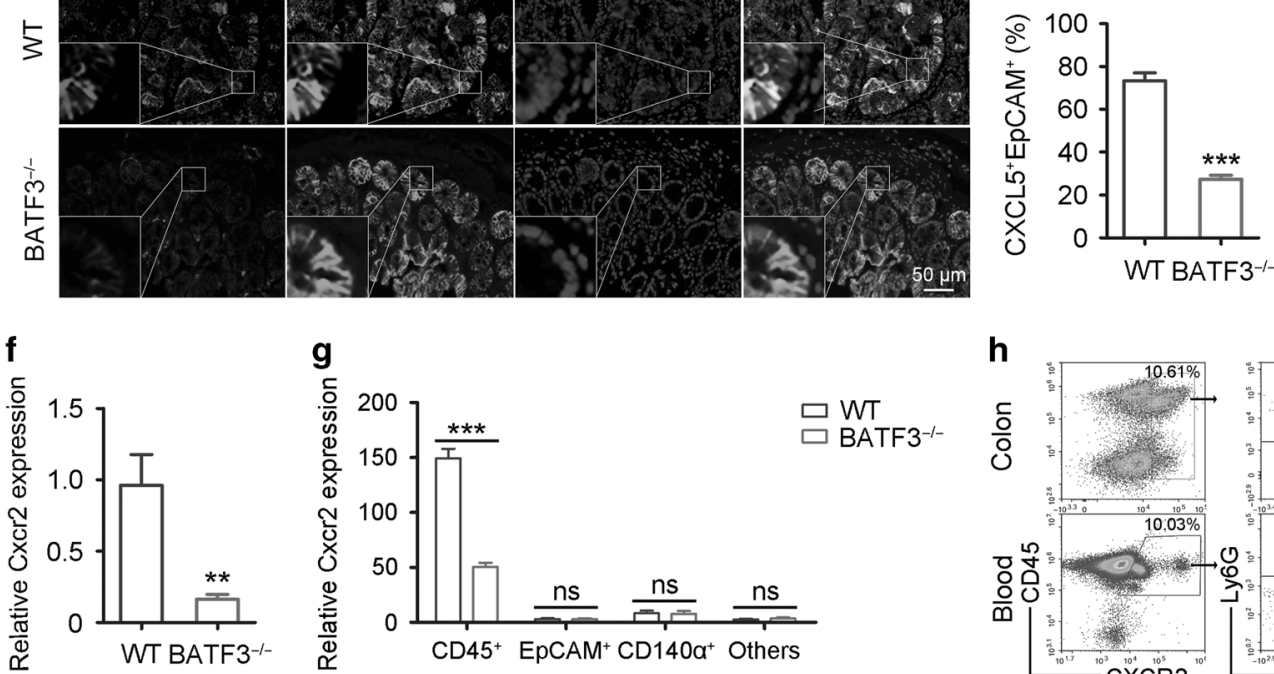

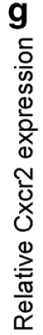
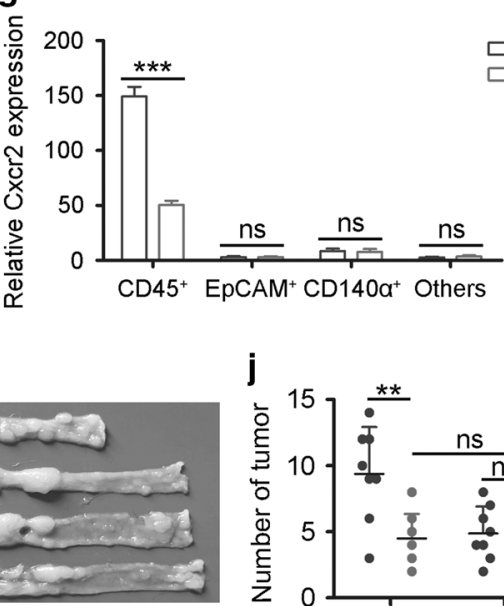

WT $\square$ BATF3 $^{-1-}$
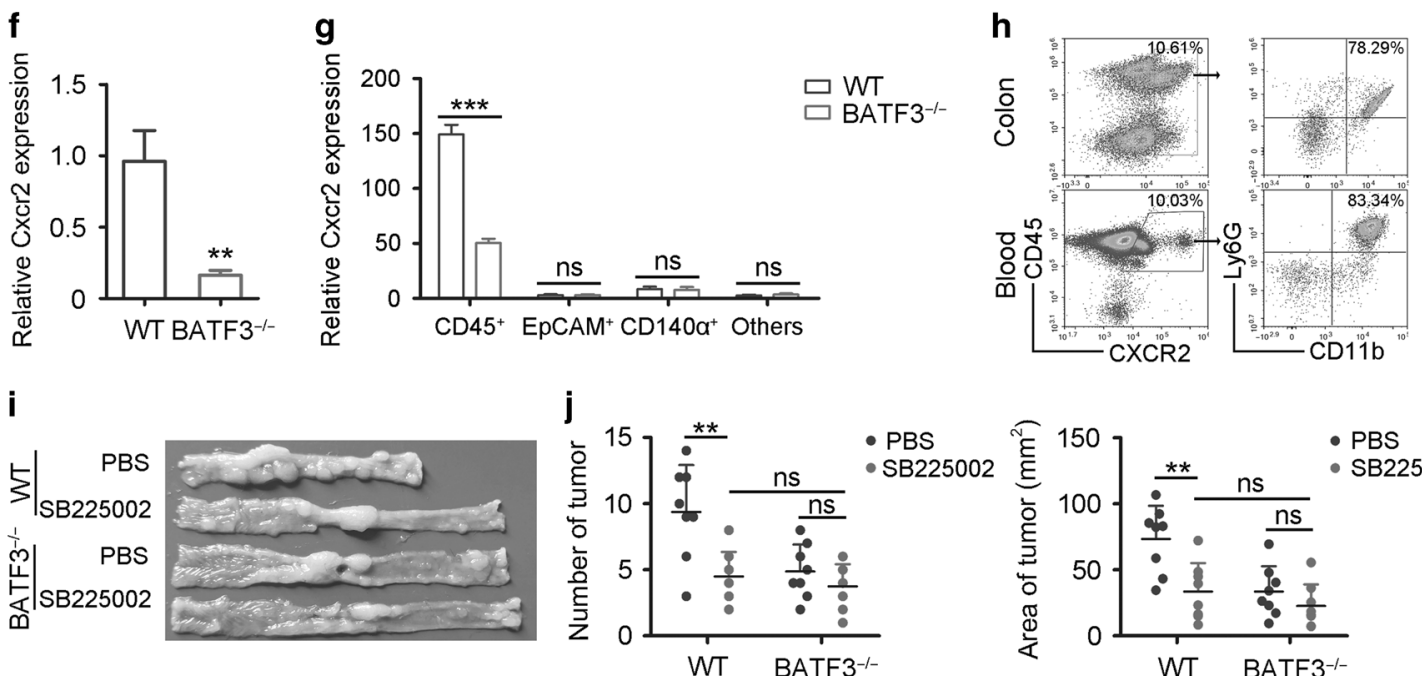

Fig. 4 BATF3 deficiency restrains CAC by inhibiting the secretion of CXCL5 in intestinal epithelial cells. a Expression of inflammatory cytokines in WT and BATF3 ${ }^{-1-}$ mouse colon tissues at the end of AOM/DSS treatment. $\mathbf{b}$ ELISA for CXCL5 expression in colon tissues from untreated or AOM/DSS-treated WT and BATF ${ }^{-1-}$ mice $(n \geq 6)$. c qPCR analysis of CXCL5 relative expression in various types of cells in colon tissues at the end of AOM/DSS treatment $(n=3)$. d qPCR analysis of CXCL5 relative expression in colon organoids from normal WT and $\mathrm{BATF}^{-1-}$ mice $(n=4)$. e Representative IF-stained images and quantification of CXCL5 and EpCAM in colon tissues at the end of AOM/DSS treatment $(n=4)$. $\mathbf{f}$ qPCR analysis of CXCR2 relative expression in colon tissues from WT and BATF3 ${ }^{-1-}$ mice at the end of AOM/DSS treatment $(n=6)$. $\mathbf{g}$ qPCR analysis of CXCR2 relative expression in various types of cells in colon tissues at the end of AOM/DSS treatment $(n=3)$. $\mathbf{h}$ Flow cytometry analysis of $\mathrm{CD}_{4} 5^{+} \mathrm{CXCR2} 2^{+}$and $\mathrm{CD} 11 \mathrm{~b}^{+} \mathrm{Ly}_{6 \mathrm{G}}{ }^{+}$cells in blood and colon tissues from WT mice at the end of AOM/DSS treatment. i Representative images of colon tissues from or vehicle (1\% DMSO in PBS) or CXCR2 inhibitors (SB225002)-injected WT and BATF3 ${ }^{-1-}$ mice at the end of AOM/DSS treatment. $\mathbf{j}$ Statistical analysis of colon tumor number and tumor area $(n=8)$. All data are representative of three independent experiments. See also Fig. S6.

To verify this, WT and BATF3 ${ }^{-1-}$ mice were injected with the CXCR2 inhibitor SB225002 $2^{32}$ to block CXCL5 recruitment of neutrophils in the AOM/DSS model. We found that blocking neutrophil recruitment with SB225002 in WT mice significantly inhibited tumor number and tumor area, but had little effect on BATF $^{-1-}$ mice (Fig. 4i, j, S6G). Taken together, these results indicate that BATF3 exacerbates CAC by promoting the secretion of CXCL5 in intestinal epithelial cells. 
BATF3 directly promotes transcription of CXCL5

Although we have shown that BATF3 promotes CXCL5 expression in intestinal epithelial cells induced by AOM/DSS, the specific molecular mechanism by which BATF3 regulates CXCL5 expression remains unclear. It has been reported that TNFa can simulate inflammatory stimulation at the cellular level, thereby promoting CXCL5 expression. ${ }^{33}$ We also found that the expression of BATF3 and CXCL5 in human intestinal epithelial cells (HIEC) and SW480 cells was significantly increased under stimulation with TNFa, meanwhile CXCL5 was the most upregulated in the case of BATF3 overexpression (Fig. 5a, b). Similarly, BATF3 knockout significantly inhibited CXCL5 expression (Fig. 5C, S7A). Furthermore, CXCL5 expression was elevated in colonic organoids stimulated with M5, but markedly downregulated in $\mathrm{BATF}^{-1-}$ intestinal organs ${ }^{34}$ (Fig. 5d). JNK, ERK, and P38 can regulate the expression of CXCL5, ${ }^{35}$ western blotting showed that the expression of $\mathrm{p}-\mathrm{JNK}, \mathrm{p}$ ERK, and p-P38 was increased under stimulation with TNFa, but the overexpression of BATF3 had little effect on these signaling pathways, thereby ruling out a possible regulatory role (Fig. S7B). As a transcription factor, BATF3 can directly bind to the promoter regions of IRF4, IRF8, MYC and other genes to promote their transcription. ${ }^{20,36}$ Whether BATF3 can directly regulate transcription of CXCL5 has not been reported, we found that BATF3 enhanced the reporter with WT CXCL5 promoter but not the mutated ones in the SW480 and HT-29 cells $^{37}$ (Fig. 5e). Through ChIP-qPCR, we further found that BATF3 could bind directly to the promoter region of the CXCL5 gene (Fig. $5 f$ ), thus confirming that BATF3 can directly regulate the transcription of CXCL5. As reported, ${ }^{38}$ BATF3 consists of 128 amino acids, the 37-62 amino acid group is the basic DNA-binding region, and the 63-91 amino acid group is the leucine zipper region. Stepwise deletion of BATF3 indicated that the amino acids $1-62$ and $63-128$ in the BATF3 fragment could not regulate the transcription of CXCL5 alone (Fig. 5g-j), In conclusion, full-length BATF3 directly promotes the transcription of CXCL5.

\section{BATF3 promotes CXCL5 transcription by forming heterodimer with} JunD

Previous studies have reported that BATF3 is an important transcription factor, which forms dimers with c-Jun, JunB, IL-9 and other proteins to generate transcriptional activity. ${ }^{12,37,39,40}$ It is unclear whether BATF3 regulates transcription of $\mathrm{CXCL} 5$ by binding to other proteins. We found that BATF3 directly combined with JunD in SW480 and HT-29 cells, as confirmed by IP-mass spectrometry, IP-WB, and IF staining (Fig. 6a-c). As for in both normal and AOM/DSS-treated colon tissues of WT mice, the BATF3 protein could also form heterodimers through binding to JunD (Fig. 6d). Stepwise deletion of BATF3 further indicated that the amino acids $1-62$ or $63-128$ of the BATF3 fragment cannot bind to JunD alone, but require full-length BATF3 (Fig. 6e). JunD knockout inhibited CXCL5 expression in SW480 and HT-29 cells (Figs. S8A, 6f, g). ChIP-qPCR further revealed that BATF3 regulated transcription of CXCL5 dependent on JunD (Fig. 6h). Collectively, we confirmed that full-length BATF3 directly promotes the transcription of CXCL5 by forming a heterodimer with JunD.

BATF3 positively correlates with CXCL5 and CD66b in human CRC tissues

To further explore the prognostic importance of BATF3 in patients with CRC, we analyzed the expression of BATF3 in human CRC Tissue microarray (TMAs). The results suggested that BATF3 expression positively correlates with tumor grade (Fig. 7a), Kaplan-Meier analysis and TCGA data analysis revealed that patients with high BATF3 expression were associated with unfavorable prognosis (Fig. 7b, c). Based on the mechanism identified above, we proceeded to explore the expression and clinical relevance between BATF3, CXCL5, and CD66b (a marker of human neutrophils) in human CRC TMAs, and we showed that the expression of BATF3, CXCL5, and CD66b were significantly increased in human malignant colon cancer tissues (Fig. 7d-f). Indeed, there were significant positive correlations between the expression of BATF3 and CXCL5, as well as BATF3 and CD66b in human colon cancer tissues (Fig. $7 \mathrm{~g}, \mathrm{~h}$ ). Furthermore, as found in the TCGA database, high expression of BATF3 also corresponded to a short survival period in tumors closely linked with inflammation, such as renal cancer, liver cancer, and stomach cancer (Fig. S9A), indicating that the BATF3 gene was closely related to inflammation and cancer. Based on the above results, we demonstrate that BATF3 in intestinal epithelial cells directly promotes the transcription of CXCL5 by forming a heterodimer with JunD, thereby recruiting neutrophils through CXCR2, and finally promoting CAC (Fig. 7i).

\section{DISCUSSION}

Previous studies have found that the BATF3 gene plays a crucial role in regulating development of $\mathrm{CDC} 1 \mathrm{~s}^{15,16}$ thereby regulating infection and tumor immunity. ${ }^{15-17,39,41}$ Although BATF3 is highly expressed in colon cancer, ${ }^{21}$ the effect of BATF3 on CAC has not yet been reported. Our results suggest that BATF3 deficiency in intestinal epithelial cells, but not in $\mathrm{CDC} 1 \mathrm{~s}$, impairs CAC mainly through inhibiting the transcriptional activity of CXCL5, resulting a blocked recruitment of $\mathrm{CXCR}^{+}$neutrophils. This important discovery will help us to broaden the narrow conception that $\mathrm{BATF}^{-1-}$ mice are equivalent to $\mathrm{CDC} 1 \mathrm{~s}$-deficient mice, meaning that the influence of the BATF3 gene on non-CDC1s under pathological conditions should also be considered.

Basic leucine zipper transcription factor ATF-like (BATF), BATF2 and BATF3 comprise the BATF family. Members of the BATF family play opposing roles in inflammation and inflammation-associated cancers. BATF2 has a low expression in CAC, and impairs colitis, and plays an important role in inhibiting angiogenesis in colon cancer. ${ }^{14,42}$ Meanwhile, BATF is highly expressed in colon cancer and promotes CAC by regulating Th17 cells. ${ }^{13}$ In the present study, we demonstrated that BATF3 is dependent on inflammatory stimulation to promote CAC in murine models. Under inflammatory stimulation, expression of BATF3 was significantly upregulated in in vitro and in vivo experiments, which further promoted the secretion of CXCL5 in intestinal epithelial cells. Various studies have clarified the regulatory role of BATF3 in the development of CDC1s. ${ }^{15,16}$ Notably, in the process of CAC, CD103 ${ }^{-}$CD $11 b^{+}$DCs increased significantly and played a major role, ${ }^{23,43}$ while the

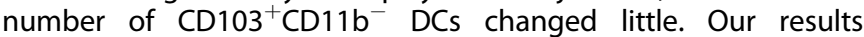
confirmed the low proportion of $\mathrm{CDC} 1 \mathrm{~s}$ (about $0.1 \%$ of $\mathrm{CD}_{4} 5^{+}$ cells) in colon tissues after CAC formed. Furthermore, $\mathrm{CDC} 1 \mathrm{~s}$ regulated by BATF3 express DNGR-1, which inhibits the functional activity of $\mathrm{CDC} 1 \mathrm{~s}^{22}$; but BATF3 deficiencies in T cells suppresses TL1A-driven mucosal inflammation. ${ }^{40} \mathrm{CDC} 1 \mathrm{~s}$ also play a limited role in tumor antigen presentation, ${ }^{39,41}$ which indicated that CDC1s had minimal effect during the development of CAC. Finally, the main upregulation of BATF3 during inflammationstimulation occurs in intestinal epithelial cells rather than in immune cells in this study. The BMT experiment further proves that promotion of colon cancer by BATF3 depends on nonimmune cells rather than on immune cells ( $C D C 1 s)$. In sum, BATF3 deficiency impaired colitis and CAC through intestinal epithelial cells, rather than $\mathrm{CDC} 1 \mathrm{~s}$.

Intestinal epithelial cells, macrophages, neutrophils, T cells, dendritic cells, and fibroblasts make up the immune microenvironment, which responds to colitis, germ infection, and tumorigenesis. ${ }^{44}$ In this context, neutrophils are significantly increased in various tumors and play an important role in promoting the development and metastasis of tumors. ${ }^{6,8}$ In our study, fewer neutrophils were observed in $\mathrm{BATF}^{-1-}$ mice under colitis and CAC. By knocking out neutrophils, we blocked the regulatory effect of BATF3 on CAC. Many studies have reported that 


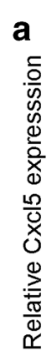
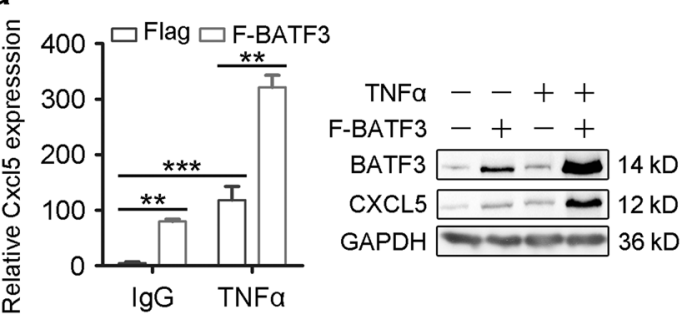

C

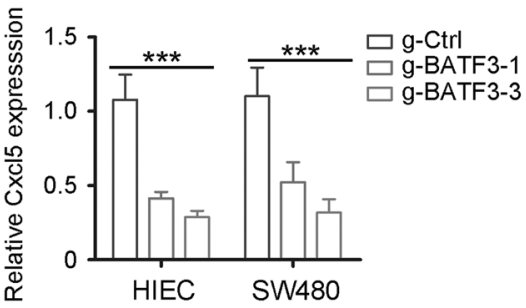

e

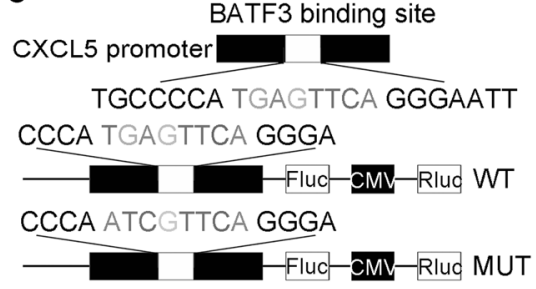

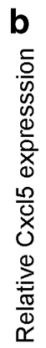

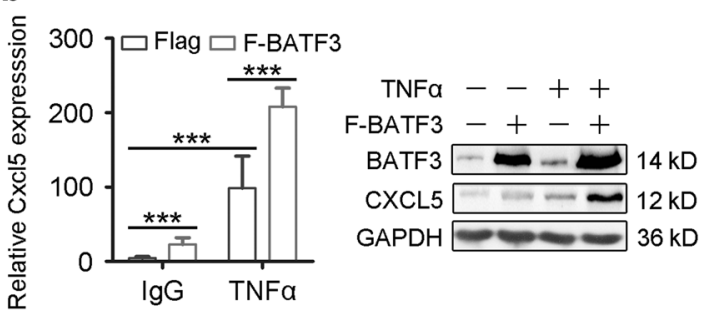

d

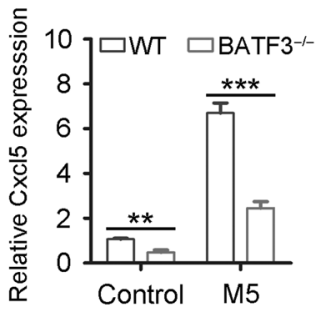

g

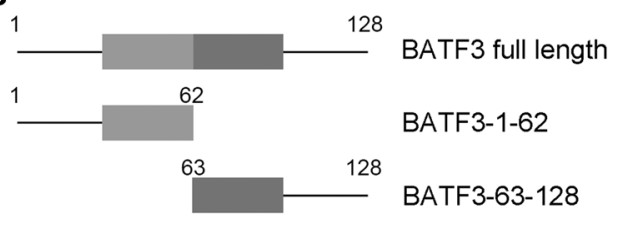

Basic DNA binding region

Leucine zipper region

i

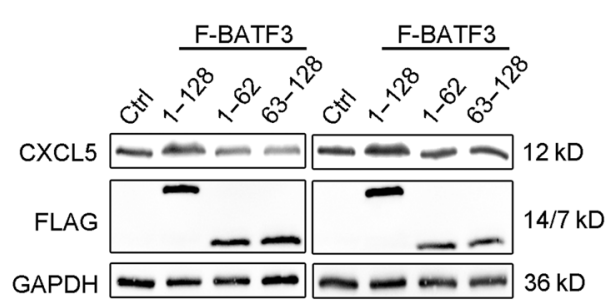

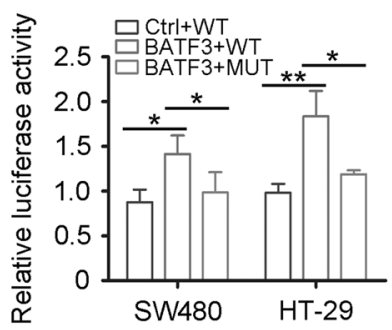

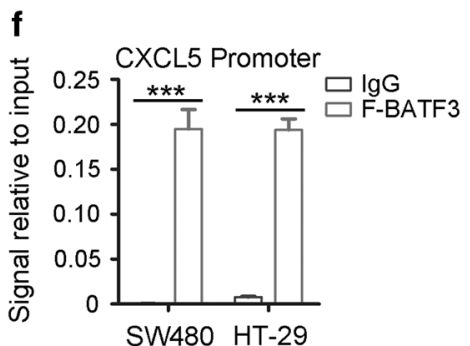

h

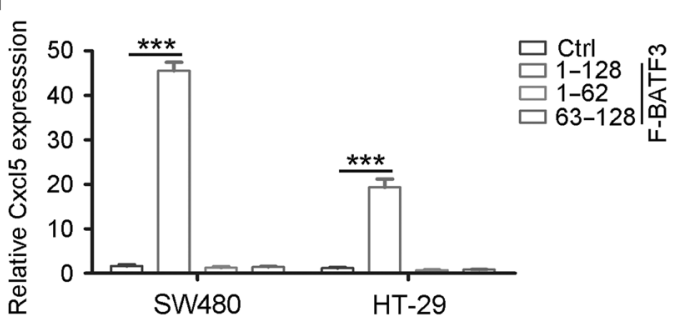

j

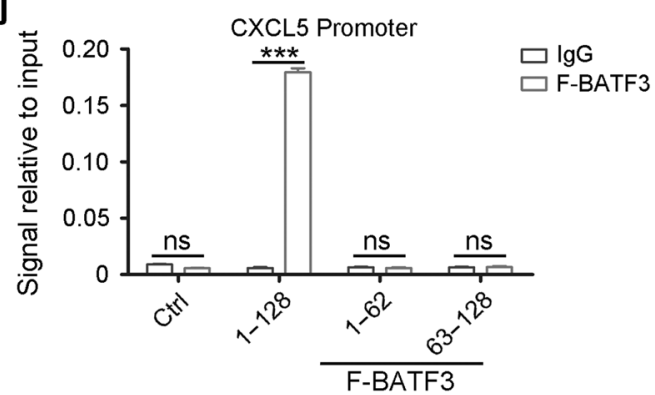

Fig. 5 BATF3 directly promotes transcription of CXCL5. qPCR and western blotting analysis of BATF3 and CXCL5 expression in HIEC (a) and SW480 (b) cells overexpressing Flag-BATF3, stimulated with $10 \mathrm{ng} / \mathrm{ml}$ TNF $\alpha$ for $48 \mathrm{~h}$. C qPCR and western blotting analysis of BATF3 and CXCL5 expression in HIEC and SW480 cells; knockout of BATF3 targeted by gRNA $(n=3)$. $\mathbf{d}$ qPCR analysis of CXCL5 relative expression in colon organoids from normal WT and BATF3 ${ }^{-1-}$ mice with M5 stimulated ( $n=3$, M5 included TNF $\alpha$, IL-1 $\beta$, IL-12p70, IL-23, and IL-6). e Schematic representation the reporter designed for determining BATF3 binding on CXCL5 loci (left), and the relative luciferase activity in SW480 and HT29 cells (right, $n=3$ ). $\mathbf{f}$ ChIP-qPCR analysis of BATF3 binding to the CXCL5 promoter in SW480 and HT-29 cells overexpressing Flag-BATF3 ( $n=$ 3). g Schematic structures of full-length BATF3, BATF3-1-62, BATF3-63-128. $\mathbf{h}$ qPCR analysis of BATF3 and CXCL5 expression in SW480 and HT29 cells $(n=3)$. i Western blotting analysis of BATF3 and CXCL5 expression in SW480 and HT-29 cells. $\mathbf{j}$ ChIP-qPCR analysis of BATF3 binding to the CXCL5 promoter in SW480 cells $(n=3)$. All data are representative of three independent experiments. See also Fig. S7. 
a

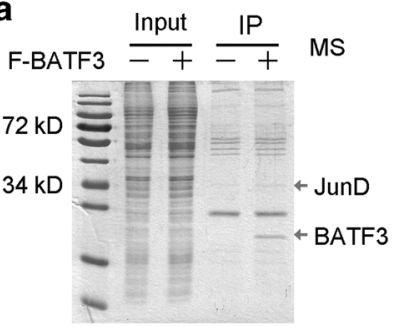

b

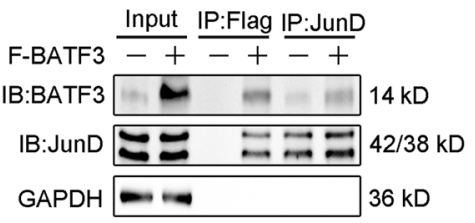

d

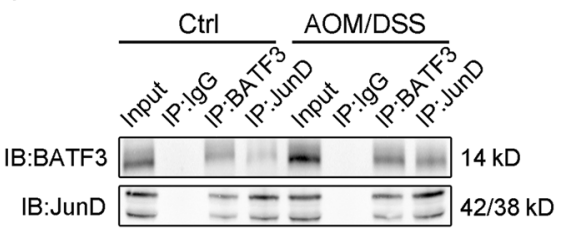

C
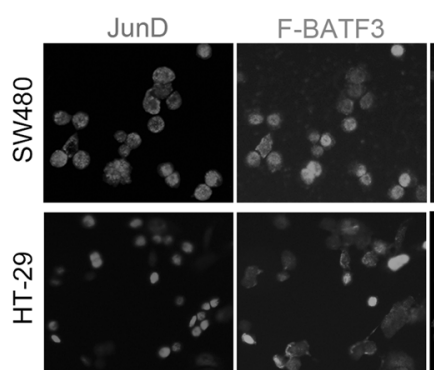

f
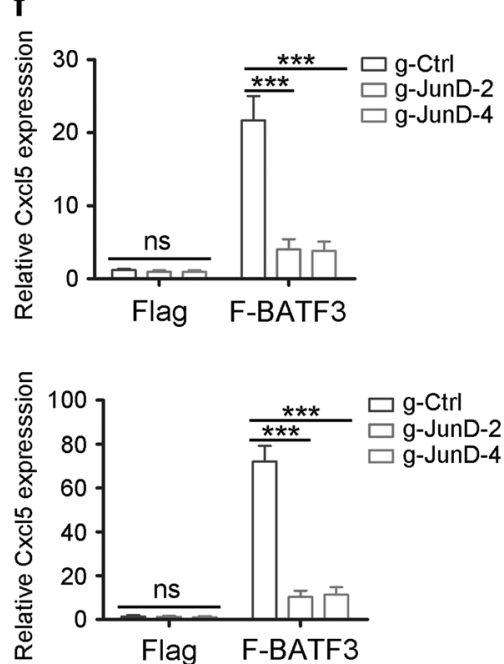

DAP1
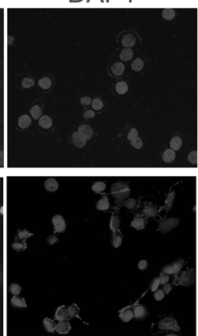

Merge
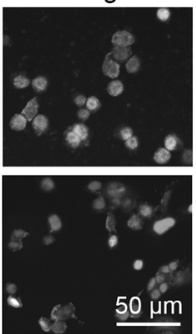

g
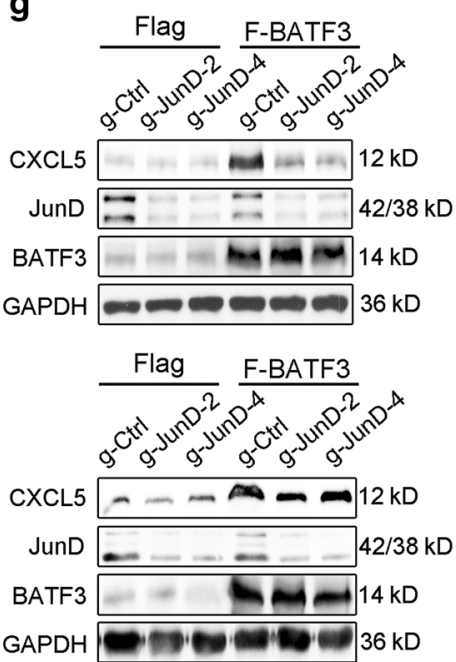

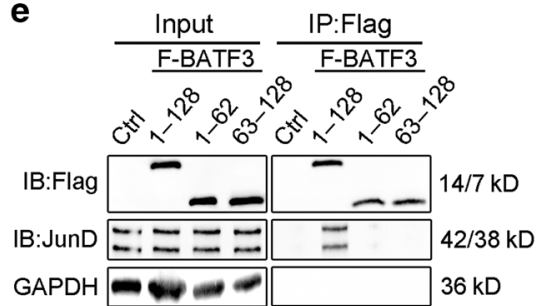

h

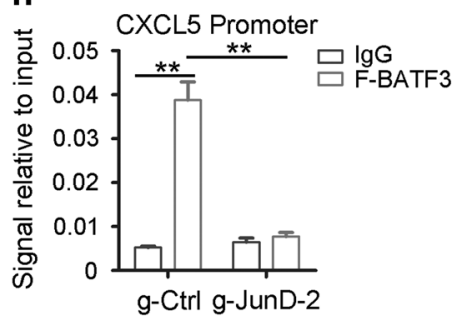

CXCL5 Promoter

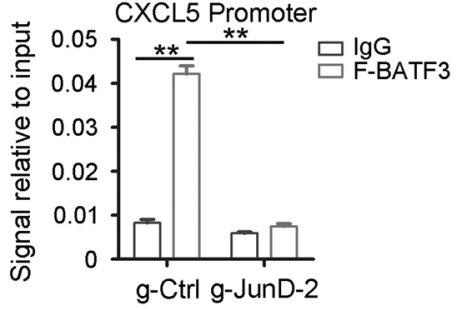

Fig. 6 BATF3 promotes CXCL5 transcription by forming heterodimer with JunD. a Coomassie blue staining of coimmunoprecipitation of SW480 cell line overexpressing Flag-BATF3. Differential bands were analyzed by mass spectrometry (MS). $\mathbf{b}$ Coimmunoprecipitation analysis of whole lysates from SW480 cells overexpressing Flag-BATF3. c Representative IF-stained images of JunD and Flag in SW480 (up) and HT-29 (down) cells overexpressing Flag-BATF3. d Coimmunoprecipitation analysis of whole lysates from in both normal and AOM/DSS-treated colon tissues of WT mice. e Coimmunoprecipitation analysis of whole lysates in SW480 cells. $\mathbf{f}$ qPCR analysis of CXCL5 relative expression in SW480 (up) and HT-29 (down) overexpressed Flag-BATF3, with JunD knockout $(n=4)$. g Western blotting analysis of CXCL5, JunD, and BATF3 expression in SW480 (up) and HT-29 (down) cells overexpressing Flag-BATF3, with JunD knockout. h ChIP-qPCR analysis of BATF3 binding to the CXCL5 promoter in SW480 (up) and HT-29 (down) overexpressing Flag-BATF3, with JunD knockout $(n=3)$. All data are representative of three independent experiments.

neutrophil recruitment is promoted by cytokines including CXCL1, CXCL2, CXCL5, and CXCL7. ${ }^{9,45}$ In our study, we demonstrated the downregulation of CXCL5 in intestinal epithelial cells from BATF $^{-l-}$ mice under inflammation conditions. Inhibition of CXCL5-CXCR2 signaling also attenuated the impairment of CAC in $\mathrm{BATF}^{-1-}$ mice. Furthermore, neutrophils can control the number and activation status of $T$ and NK cells. ${ }^{11}$ We found increased $\mathrm{CD}^{+}{ }^{+} \mathrm{FNY}^{+} \mathrm{T}$ cell and decreased IL17A ${ }^{+} \mathrm{CD} 4^{+} \mathrm{T}$ cell counts in colon cancer tissues of BATF $3^{-1-}$ mice; this result was consistent with the antitumor effect of BATF3 deficiency. It is known that BATF promotes CAC by promoting Th17 cells. ${ }^{13}$ We also found that BATF significantly increased in colon tissues of AOM/DSS-treated mice, but BATF3 gene knockout did not affect the expression of BATF. Thus, we speculated that the promoting role of BATF3 on CAC is BATF independent (data not shown). Thus, our results indicate that neutrophils recruited by intestinal epithelial BATF3 may promote colon cancer by regulating these $\mathrm{T}$ cells, although the detailed mechanism needs to be further investigated.

BATF3 belongs to the AP-1 superfamily. As a transcription factor, BATF3 forms dimers with c-Jun, JunB, IL-9 and other proteins to generate transcriptional activity. ${ }^{12,37,40}$ The results by Co-IP-mass spectrometry and subsequent IP-WB verification suggest that BATF3 directly combines with JunD. JunD knockout inhibited CXCL5 expression. On the other hand, BATF3 can directly bind to the promoter region of IRF4, IRF8, MYC and other genes to promote their transcription. ${ }^{20,36}$ Through ChIP-qPCR, we found that BATF3 can bind directly to the promoter region of the CXCL5 gene, thereby directly regulating transcription of CXCL5 in the presence of JunD. Thus, we addressed the fact that BATF3 directly promotes the transcription of CXCL5 by binding to JunD to form a dimer. 
$Y$ Lin et al.

a

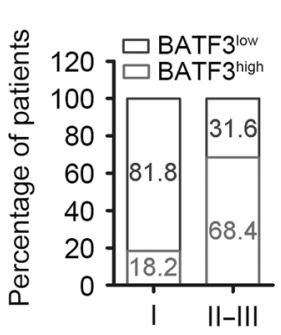

d
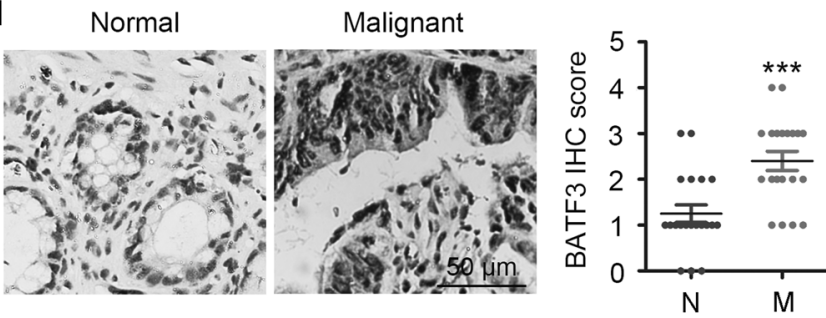

e
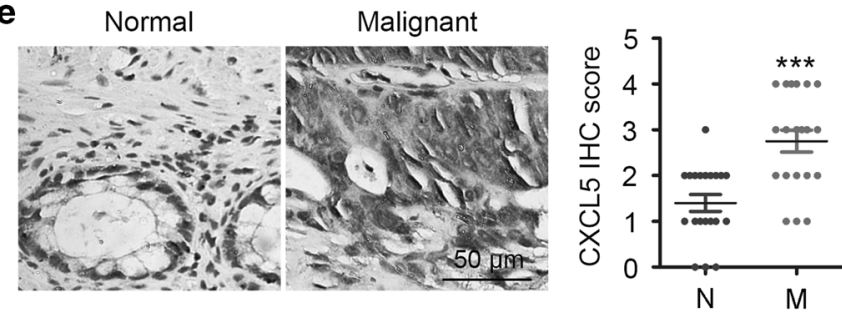

f
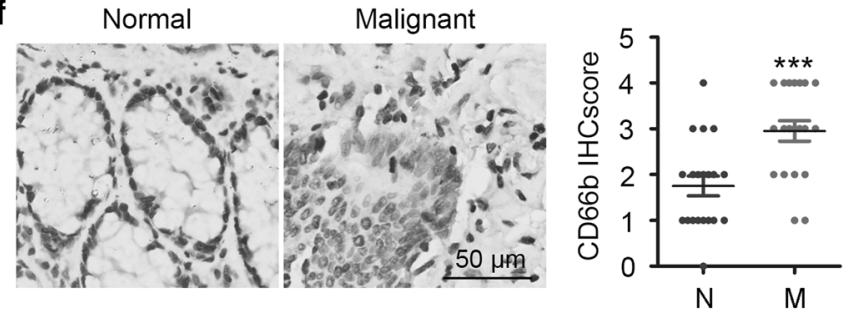

C

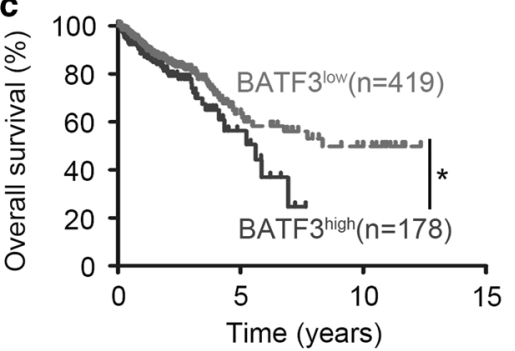

9

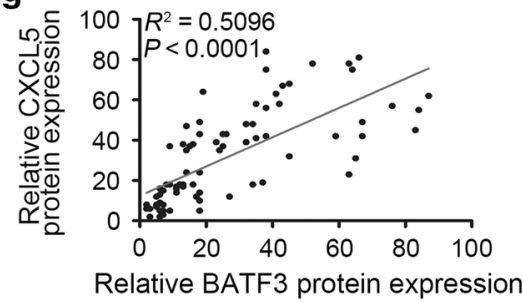

h

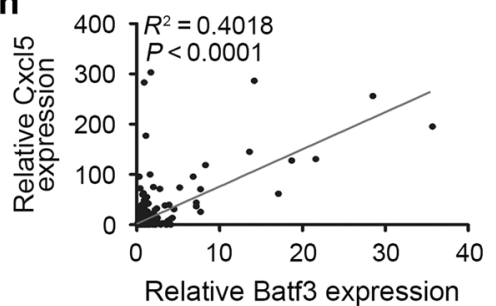

i

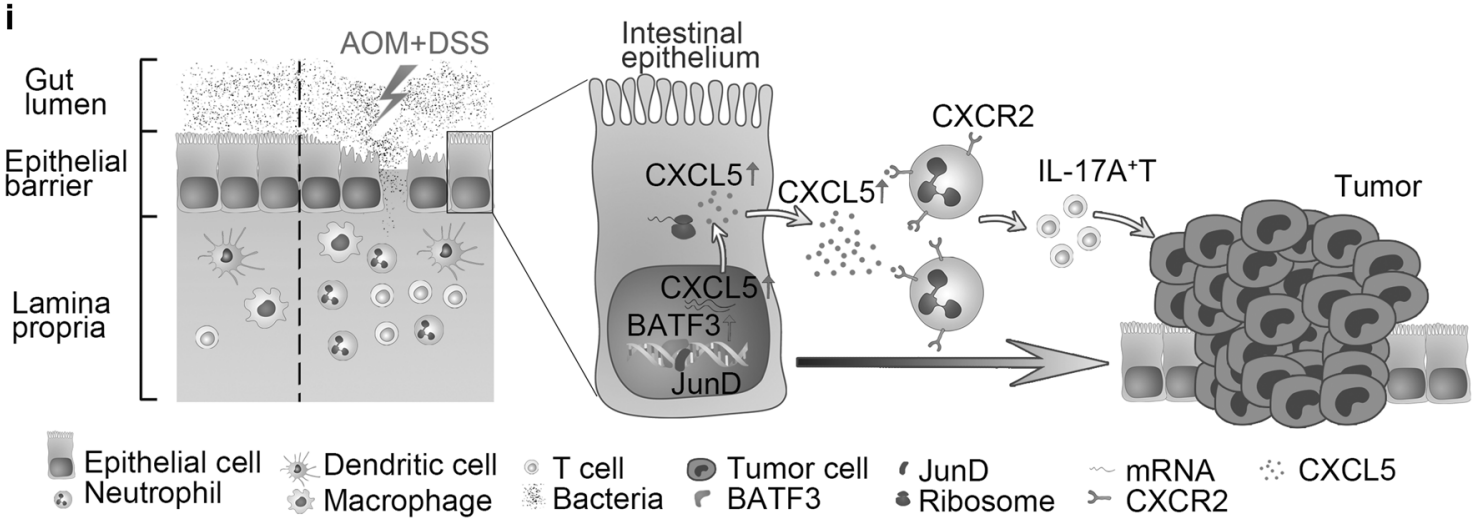

Fig. 7 BATF3 positively correlates with CXCL5 and CD66b in human CRC tissues. a Percentage of patients with high or low expression of BATF3 according to tumor stage, numbers in bars represent the percentage of patients. b Kaplan-Meier curve shows overall survival of patients with colon cancer, stratified by BATF3 expression (log-rank test). c Overall survival curve of patients with colorectal cancer, stratified by BATF3 expression (website of TCGA RNA-seq data: https://www.proteinatlas.org/ENSG00000 123685-BATF3/pathology/tissue/ colorectal+cancer). Representative images and quantification of BATF3 (d), CXCL5 (e) and CD66b (f) via IHC staining of human normal and malignant colon tissues $(n=20$, Mann-Whitney test). $\mathbf{g}$ Statistical analysis of the correlation between BATF3 and CXCL5, as well as BATF3 and CD66b in colon cancer TMAs ( $n=85$, linear regression). $\mathbf{h}$ Correlation analysis of TCGA RNA-seq data for BATF3 and CXCL5 expression across colorectal cancer samples $(n=595$, Pearson correlation coefficients, website of TCGA RNA-seq data: https://www.proteinatlas.org/ENSG0000 123685-BATF3/pathology/tissue/colorectal+cancer). i Schematic representation of the BATF3-CXCL5-neutrophil axis in colitis-associated colon cancer. See also Fig. S8. 
BATF3 is an oncogene in CRC and glioma, and also predicts the poor prognosis of patients. ${ }^{19,20}$ Our results confirm the upregulation of BATF3 in CRC tissues from patients and the correlation between BATF3 expression and poor prognosis of CRC patients. CXCL5 has also been shown to be a predictor of poor prognosis in $\mathrm{CRC}^{46}$ while aggregation of neutrophils often correlates with shorter survival time in CRC patients. ${ }^{47}$ The results of IHC staining in CRC TMAs confirmed the positive correlation between BATF3 and CXCL5 expression, as well as BATF3 and CD66b expression. These findings were consistent with those of mechanistic investigations, providing further evidence of the predictive role of BATF3 in the prognosis of patients. In addition, TCGA database analysis showed that high expression of BATF3 was negatively correlated with survival of $\mathrm{CRC}$, renal cancer, liver cancer, and stomach cancer, which are closely related to inflammation, indicating that the BATF3 gene may play a significant role in promoting several inflammationrelated cancers, and can be used as a potential target for clinical diagnosis of CRC.

In summary, under inflammatory stimulation, intestinal epithelial BATF3 directly promotes the transcription of CXCL5 by forming a heterodimer with JunD, thereby recruiting neutrophils through CXCR2, regulating $T$ cells, and finally promoting CAC. The novel findings in our study suggest that BATF3 may be a novel diagnostic indicator for $\mathrm{CAC}$, and possibly for other inflammationassociated cancers, as well.

\section{MATERIALS AND METHODS}

\section{Clinical samples}

TMAs of human malignant colon tissues and their adjacent normal tissues were purchased from Shanghai Outdo Biotech. Co. Ltd (HColA180Su10, China). Additional human malignant colon tissues and some adjacent normal tissues were obtained from the clinical sample database of West China Hospital (Chengdu, China).

\section{Mice and cell lines}

The BATF3 wild-type (C57BL/6J, 000664), BATF3-deficient (B6.129S (C)-Batf3tm $1 \mathrm{Kmm} / \mathrm{J}, 013755)$ mice were purchased from Jackson Laboratory (USA). Normal CD45.1 mice were obtained from Prof Chong Chen (Sichuan University). HEK293T cells, HIEC, human colon cancer cells SW480, and HT-29 were purchased from the American Type Culture Collection (USA). All of the cell lines have been confirmed as mycoplasma contamination free with the kit from Yise Medical Technology (PM008, China).

\section{Animal models}

Animal models were established in a previous study. ${ }^{24}$ Briefly, to create the CAC model, mice were injected intraperitoneally (i.p.) with $10 \mathrm{mg} / \mathrm{kg}$ AOM (Sigma, A5486, Germany). After 7 days, 2\% DSS (MP Biologicals, USA) was given in drinking water for 7 days, followed by regular drinking water for 14 days. This cycle was repeated twice, and mice were sacrificed after the last DSS cycle. To create the model of colitis-independent colon cancer, mice received an injection of $10 \mathrm{mg} / \mathrm{kg}$ of AOM for 6 weeks and were sacrificed 25 weeks after the last injection. The degree of tumor formation and colitis was observed by high-resolution miniendoscopy (KARL STOKZ, Germany). Scoring for stool consistency and occult blood was performed as previously described. ${ }^{24}$ Double-blind histological assessments of colitis and severity scores were given after hematoxylin and eosin (H\&E) staining as described. ${ }^{48}$

Bone marrow cross-transfusion (BMT)

Cell suspensions from male WT (CD45.1) and BATF3 ${ }^{-1-}$ (CD45.2) mouse bone marrow were prepared. The WT and BATF3 ${ }^{-1-}$ male mice were $\gamma$-irradiated with 8 Gray using the RS-200 Biological Irradiator (Rad-Source Technologies, USA), and then received a single intravenous injection of $1 \times 10^{7}$ bone marrow cells. Two months after BM reconstitution, mice were used for CAC experiments. $^{49}$

Flow cytometry analysis and sorting

Colonic lamina propria immunocytes were isolated according to a previous report. ${ }^{50}$ Fixable Viability Stain 620 (BD Biosciences, USA) was used to discriminate live cells. We then blocked with Fc-block (BD Biosciences, USA) and stained using antibodies. Data were acquired and analyzed on a NovoCyte flow cytometer (ACEA Biosciences, USA). Labeled cells were sorted on a flow cell sorter (BD: FACSAria SORP). All flow antibodies are listed in Supplemental information.

Chromatin immunoprecipitation (ChIP)

Instructions for the Simple ChIP ${ }^{\circledR}$ Plus Enzymatic Chromatin IP Kit (CST, 9005, USA) were followed. In brief, cells were crosslinked with $4 \%$ formaldehyde followed by cell collection and enzymatic digestion to yield genomic DNA fragments $150-900 \mathrm{bp}$. Chromatin was immunoprecipitated using an anti-histone $\mathrm{H} 3$ antibody (CST, 4620, USA), or anti-Flag antibody (CST, 14793, USA).

Statistical analysis

Data were analyzed using GraphPad Prism 5 software. Results are expressed as means \pm standard error of the mean (SEM). Statistical significance between groups was determined using the Student's $t$ test or one-way ANOVA. Survival analysis was presented using the Kaplan-Meier survival estimate. In the figures, symbols used to denote significance are as follows: ${ }^{*} P<0.05,{ }^{*} P<0.01,{ }^{* *} P<$ 0.001 , and ns no statistical significance.

\section{ACKNOWLEDGEMENTS}

This work was supported by grants from the National Natural Science Foundation of China (Nos. 81773050, 81772939, 81972607) and 1.3 .5 project for disciplines of excellence, West China Hospital, Sichuan University. We are grateful to Prof Chong Chen for offering CD45.1 mice.

\section{AUTHOR CONTRIBUTIONS}

Y.L. and L.C. performed most of the experiments with assistance from Y.L., and Y.W.H. D., M.J. and L.D. were involved in obtaining funding, study concept design, analysis and interpretation of data, drafting of the manuscript and critical revision of the manuscript for important intellectual content. X.H., C.F., and Z.G.Z. were involved in collecting human colon cancer tissues. Q.W., H.L.W., G.S., J.S.L., Q.W., Q.M.Y., S.C. and X.L.S. were involved in animal study. Y.Y. and P.F. were involved in technical support and analysis and interpretation of data.

\section{ADDITIONAL INFORMATION}

The online version of this article (https://doi.org/10.1038/s41385-020-0297-3) contains supplementary material, which is available to authorized users.

Competing interests: The authors declare no competing interests.

Publisher's note Springer Nature remains neutral with regard to jurisdictional claims in published maps and institutional affiliations.

\section{REFERENCES}

1. Zauber, A. G. et al. Colonoscopic polypectomy and long-term prevention of colorectal-cancer deaths. N. Engl. J. Med. 366, 687-696 (2012).

2. Siegel, R. L., Miller, K. D. \& Jemal, A. Cancer statistics, 2020. CA Cancer J. Clin. 70, 7-30 (2020).

3. Brody, H. Colorectal cancer. Nature 521, S1 (2015).

4. Greten, F. R. \& Grivennikov, S. I. Inflammation and cancer: triggers, mechanisms, and consequences. Immunity 51, 27-41 (2019).

5. Coussens, L. M., Zitvogel, L. \& Palucka, A. K. Neutralizing tumor-promoting chronic inflammation: a magic bullet? Science 339, 286-291 (2013).

6. Coffelt, S. B., Wellenstein, M. D. \& de Visser, K. E. Neutrophils in cancer: neutral no more. Nat. Rev. Cancer 16, 431-446 (2016). 
7. Shaul, M. E. \& Fridlender, Z. G. Tumour-associated neutrophils in patients with cancer. Nat. Rev. Clin. Oncol. 16, 601-620 (2019).

8. Kvedaraite, E. et al. Tissue-infiltrating neutrophils represent the main source of IL23 in the colon of patients with IBD. Gut 65, 1632-1641 (2016).

9. Katoh, H. et al. CXCR2-expressing myeloid-derived suppressor cells are essential to promote colitis-associated tumorigenesis. Cancer Cell 24, 631-644 (2013).

10. Yu, P. F. et al. TNFalpha-activated mesenchymal stromal cells promote breast cancer metastasis by recruiting CXCR2(+) neutrophils. Oncogene 36, 482-490 (2017).

11. Gabrilovich, D. I. \& Nagaraj, S. Myeloid-derived suppressor cells as regulators of the immune system. Nat. Rev. Immunol. 9, 162-174 (2009).

12. Bower, K. E., Zeller, R. W., Wachsman, W., Martinez, T. \& McGuire, K. L. Correlation of transcriptional repression by p21(SNFT) with changes in DNA.NF-AT complex interactions. J. Biol. Chem. 277, 34967-34977 (2002).

13. Punkenburg, E. et al. Batf-dependent Th17 cells critically regulate IL-23 driven colitis-associated colon cancer. Gut 65, 1139-1150 (2016).

14. Dai, L. et al. SARI inhibits angiogenesis and tumour growth of human colon cancer through directly targeting ceruloplasmin. Nat. Commun. 7, 11996 (2016).

15. Hildner, K. et al. Batf3 deficiency reveals a critical role for CD8alpha+ dendritic cells in cytotoxic T cell immunity. Science 322, 1097-1100 (2008).

16. Edelson, B. T. et al. Peripheral CD103+ dendritic cells form a unified subset developmentally related to CD8alpha+ conventional dendritic cells. J. Exp. Med. 207, 823-836 (2010)

17. Mashayekhi, M. et al. CD8alpha(+) dendritic cells are the critical source of interleukin-12 that controls acute infection by Toxoplasma gondii tachyzoites. Immunity 35, 249-259 (2011).

18. Kuehlwein, J. M. et al. Protection of Batf3-deficient mice from experimental cerebral malaria correlates with impaired cytotoxic T-cell responses and immune regulation. Immunology 159, 193-204 (2019).

19. Vrzalikova, K. et al. S1PR1 drives a feedforward signalling loop to regulate BATF3 and the transcriptional programme of Hodgkin lymphoma cells. Leukemia 32, 214-223 (2018).

20. Lollies, A. et al. An oncogenic axis of STAT-mediated BATF3 upregulation causing MYC activity in classical Hodgkin lymphoma and anaplastic large cell lymphoma. Leukemia 32, 92-101 (2018).

21. Cao, L. et al. MiR-760 suppresses human colorectal cancer growth by targeting BATF3/AP-1/cyclinD1 signaling. J. Exp. Clin. Cancer Res. 37, 83 (2018).

22. Del Fresno, $C$. et al. DNGR-1 in dendritic cells limits tissue damage by dampening neutrophil recruitment. Science 362, 351-356 (2018).

23. Varol, C. et al. Intestinal lamina propria dendritic cell subsets have different origin and functions. Immunity 31, 502-512 (2009).

24. Neufert, C., Becker, C. \& Neurath, M. F. An inducible mouse model of colon carcinogenesis for the analysis of sporadic and inflammation-driven tumor progression. Nat. Protoc. 2, 1998-2004 (2007).

25. Chao, T., Furth, E. E. \& Vonderheide, R. H. CXCR2-dependent accumulation of tumor-associated neutrophils regulates T-cell immunity in pancreatic ductal adenocarcinoma. Cancer Immunol. Res. 4, 968-982 (2016).

26. Steele, C. W. et al. CXCR2 inhibition profoundly suppresses metastases and augments immunotherapy in pancreatic ductal adenocarcinoma. Cancer Cell 29, 832-845 (2016)

27. Moynihan, K. D. et al. Eradication of large established tumors in mice by combination immunotherapy that engages innate and adaptive immune responses. Nat. Med. 22, 1402-1410 (2016).

28. Wang, G. et al. Targeting YAP-dependent MDSC infiltration impairs tumor progression. Cancer Discov. 6, 80-95 (2016).

29. Mei, J. et al. CXCL5 regulates chemokine scavenging and pulmonary host defense to bacterial infection. Immunity 33, 106-117 (2010).
30. Murdoch, C., Muthana, M., Coffelt, S. B. \& Lewis, C. E. The role of myeloid cells in the promotion of tumour angiogenesis. Nat. Rev. Cancer 8, 618-631 (2008).

31. Le, Y., Zhou, Y., Iribarren, P. \& Wang, J. Chemokines and chemokine receptors: their manifold roles in homeostasis and disease. Cell. Mol. Immunol. 1, 95-104 (2004).

32. Kumar, V. et al. Cancer-associated fibroblasts neutralize the anti-tumor effect of CSF1 receptor blockade by inducing PMN-MDSC infiltration of tumors. Cancer Cell 32, 654-668 e655 (2017).

33. Madorin, W. S. et al. Cardiac myocytes activated by septic plasma promote neutrophil transendothelial migration: role of platelet-activating factor and the chemokines LIX and KC. Circulation Res. 94, 944-951 (2004).

34. Bhattacharya, $\mathrm{N}$. et al. Normalizing microbiota-induced retinoic acid deficiency stimulates protective $\mathrm{CD} 8(+) \mathrm{T}$ cell-mediated immunity in colorectal cancer. Immunity 45, 641-655 (2016).

35. Xia, J., Xu, X., Huang, P., He, M. \& Wang, X. The potential of CXCL5 as a target for liver cancer - what do we know so far? Expert Opin. Ther. Targets 19, 141-146 (2015).

36. Grajales-Reyes, G. E. et al. Batf3 maintains autoactivation of Irf8 for commitment of a CD8alpha(+) conventional DC clonogenic progenitor. Nat. Immunol. 16, 708-717 (2015)

37. lacobelli, M., Wachsman, W. \& McGuire, K. L. Repression of IL-2 promoter activity by the novel basic leucine zipper p21SNFT protein. J. Immunol. 165, 860-868 (2000).

38. Lu, S. et al. CDD/SPARCLE: the conserved domain database in 2020. Nucleic Acids Res. 48(D1), D265-D268 (2020).

39. Salmon, $H$. et al. Expansion and activation of $\mathrm{CD} 103(+)$ dendritic cell progenitors at the tumor site enhances tumor responses to therapeutic PD-L1 and BRAF inhibition. Immunity 44, 924-938 (2016).

40. Tsuda, M. et al. A role for BATF3 in TH9 differentiation and T-cell-driven mucosal pathologies. Mucosal Immunol. 12, 644-655 (2019).

41. Sanchez-Paulete, A. R. et al. Cancer immunotherapy with immunomodulatory anti-CD137 and anti-PD-1 monoclonal antibodies requires BATF3-dependent dendritic cells. Cancer Discov. 6, 71-79 (2016).

42. Su, Z. Z. et al. Cloning and characterization of SARI (suppressor of AP-1, regulated by IFN). Proc. Natl Acad. Sci. USA 105, 20906-20911 (2008).

43. Arnold, I. C. et al. CD11c(+) monocyte/macrophages promote chronic Helicobacter hepaticus-induced intestinal inflammation through the production of IL23. Mucosal Immunol. 9, 352-363 (2016).

44. Nowarski, R., Jackson, R. \& Flavell, R. A. The stromal intervention: regulation of immunity and inflammation at the epithelial-mesenchymal barrier. Cell 168, 362-375 (2017).

45. Rot, A. \& von Andrian, U. H. Chemokines in innate and adaptive host defense: basic chemokinese grammar for immune cells. Annu. Rev. Immunol. 22, 891-928 (2004).

46. Yildirim, K. et al. Clinical value of CXCL5 for determining of colorectal cancer. Asian Pac. J. Cancer Prev. 19, 2481-2484 (2018).

47. Li, T. J. et al. Interleukin-17-producing neutrophils link inflammatory stimuli to disease progression by promoting angiogenesis in gastric cancer. Clin. Cancer Res. 23, 1575-1585 (2017).

48. Williams, K. L. et al. Enhanced survival and mucosal repair after dextran sodium sulfate-induced colitis in transgenic mice that overexpress growth hormone. Gastroenterology 120, 925-937 (2001).

49. Xiao, Z. et al. The pivotal role of IKKalpha in the development of spontaneous lung squamous cell carcinomas. Cancer Cell 23, 527-540 (2013).

50. Katlinski, K. V. et al. Inactivation of interferon receptor promotes the establishment of immune privileged tumor microenvironment. Cancer Cell 31, 194-207 (2017). 